\title{
DESCRIZIONE PETROGRAFICA DI CERAMICHE COMUNI DI ETÀ ROMANA DA SAN POTITO IN BASE A SEZIONI SOTTILI
}

\author{
GYÖRGY SZAKMÁNY - DÓRA KÜRTHY
}

\author{
Cattedra di Petrografia e Geochimica dell'Università Loránd Eötvös di Budapest \\ Pázmány sétány $1 / \mathrm{c}, \mathrm{H}-1117$ Budapest, Ungheria \\ E-mail: gyorgy.szakmany@geology.elte.hu, dora.kurthy@gmail.com
}

\begin{abstract}
Twenty Roman Age home-made sherds from Central Italian San Potito locality were studied by petrographic microscopic method. The ceramics were divided into five petrographic groups on the basis of their composition and structural-textural features. Two groups of the ceramics were tempered with clasts of alkaline volcanic origin, which seem to originate from CentralItalian volcanic territory components. The ceramics belonging to the other three groups contained large amounts of limestone and carbonatic fossils, the origin of the raw material was a marine clayish sediment, perhaps flysch.
\end{abstract}

Keywords: Roman Age, ceramic, petrography, alkali volcanic temper

\section{INTRODUZIONE}

Si descrivono qui di seguito i risultati dell'analisi petrografica di venti frammenti di ceramica comune, provenienti dallo scavo del sito centro italico di San Potito.

Dopo la descrizione macroscopica delle ceramiche da analizzare si è passati alla preparazione di sezioni sottili coperte dello spessore di $30 \mu \mathrm{m}$, perpendicolari rispetto alla parete delle ceramiche, per sottoporle in seguito ad un'analisi eseguita con microscopio petrografico (polarizzatore) e nel corso delle analisi, si sono eseguite le valutazioni qualitative e semiqualitative dei costituenti presenti nelle ceramiche con la scala Milner-Watts a nove gradi, portando a termine, inoltre, una dettagliata caratterizzazione tessiturale. Il nostro lavoro si basa sulla metodologia elaborata da Szakmány ${ }^{1}$, ma abbiamo preso in considerazione anche le indicazioni espresse dal Prehistoric Ceramics Research Group, ${ }^{2}$ nonché i lavori di Whitbread ${ }^{3}$, e Quinn ${ }^{4}$ relativi alla descrizione petrografica delle ceramiche.

Nella descrizione dei granuli del degrassante, abbiamo preso in considerazione le opere di Pettijohn e altri ${ }^{5}$ e di Tucker6.

I risultati sono esposti separatamente per ogni gruppo di ceramiche, nell'ordine seguente: per primo abbiamo presentato le caratteristiche macroscopiche, poi le caratteristiche tessiturali che si vedono al microscopio, quindi i costituenti aplastici del materiale (granuli, frammenti, materiale smagrante).

In base alle similitudini e differenze dei costituenti aplastici, nonché alle caratteristiche tessiturali delle ceramiche il materiale analizzato è stato inquadrato in cinque gruppi ed entro il gruppo I abbiamo formato ulteriori sottogruppi.

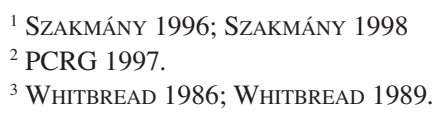

${ }^{3}$ WhitBREAD 1986; WhItBREAD 1989.

\footnotetext{
${ }^{4}$ QUinN 2013.

${ }^{5}$ PetTIJohn e altri 1972

${ }^{6}$ TUCKER 2001.
} 
I gruppi formati definitivamente e il numero dei campioni sono i seguenti:

Gruppo I: I/a): SP-2, SP-3, SP-7, SP-8, SP-12, SP-15, SP-17, SP-18

$\mathrm{I} / \mathrm{b}): \mathrm{SP}-6, \mathrm{SP}-14$

I (generico): SP-13 (il campione era di dimensioni molto piccole quindi non si è potuto decidere se appartenesse al sottogruppo /a) o /b)

Gruppo II: SP-5, SP-11, SP-16, SP-20, SP-21

Gruppo III: SP-10, SP-19

Gruppo IV: SP-1

Gruppo V: SP-9

2 RISULTATI DELLE ANALISI

\section{Gruppo I}

Questo gruppo è costituito da undici campioni, divisi in due sottogruppi (I / a, I / b); le dimensioni troppo piccole di uno dei campioni, non ci hanno permesso di raccogliere dalla sezione sottile il numero di informazioni richiesto per collocarlo in uno dei sottogruppi, perciò l'abbiamo inquadrato nel I gruppo avente l'indicazione di "generico".

I/a): SP-2, SP-3, SP-7, SP-8, SP-12, SP-15, SP-17, SP-18

I/b): SP-6, SP-14

I (generico): SP-13

\section{Descrizione macroscopica}

Quasi ogni impasto del gruppo I è caratteristicamente di color rosso-mattone, con granuli neri, bianchi e individui di mica bianca sparsi visibili a occhio nudo. La struttura del campione SP-14 è a sandwich, il nucleo è grigio e la parte esterna di color rosso-mattone opaco, ma similmente alle altre ceramiche anche in questa risaltano i costituenti sparsi, di color nero, bianco e argento.

\section{Descrizione microscopica}

\section{Gruppo I/a}

Tessitura

In base alla granulometria dei costituenti aplastici le ceramiche possono essere definite di tessitura iatale o leggermente iatale, in genere la distribuzione ha 3 massimali, e due campioni (SP- 15, SP-18) hanno 2 massimali (distribuzione bimodale). La granulometria dominante della frazione più fine dei costituenti aplastici in generale è pari a 20-50 $\mu \mathrm{m}$ (raramente raggiungono gli $80 \mu \mathrm{m}$ ), la granulometria media è pari generalmente a 100-150 (80-200) $\mu \mathrm{m}$, e la frazione più grossolana in genere è di 300-600 $(250-800) \mu \mathrm{m}$, mentre la dimensione massima rilevata nei relativi campioni varia da $\mathbf{7 0 0}$ a $1500 \mu \mathrm{m}$. Le ceramiche campionate sono generalmente poco assortite, salvo due, (SP-15, SP-18) che sono mediamente assortite. I granuli generalmente non sono per niente arrotondati o solo poco arrotondati e molti sono i grani scheggiati. Sporadicamente si trovano anche granuli mediamente arrotondati.

La percentuale dei costituenti aplastici di dimensioni più grandi di $15 \mu \mathrm{m}$ è circa del $25 \%$, si rileva una percentuale minore nei campioni SP-15 e SP-18, nel primo 15 e nel secondo il $18 \%$, mentre nel campione SP-17 (nonché in quello SP-14 appartenente al gruppo I / b) è leggermente più alta, raggiungendo circa il $30 \%$.

Le ceramiche sono generalmente compatte e contengono alcuni macro-pori di forma irregolare (le dimensioni sono generalmente di $250-800 \mu \mathrm{m}$, ma vi si trovano anche pori più grandi che possono arrivare anche a 2000 $\mu \mathrm{m})$. Nei campioni SP-15 e SP-18 i pori sono quasi esclusivamente di piccole dimensioni. 

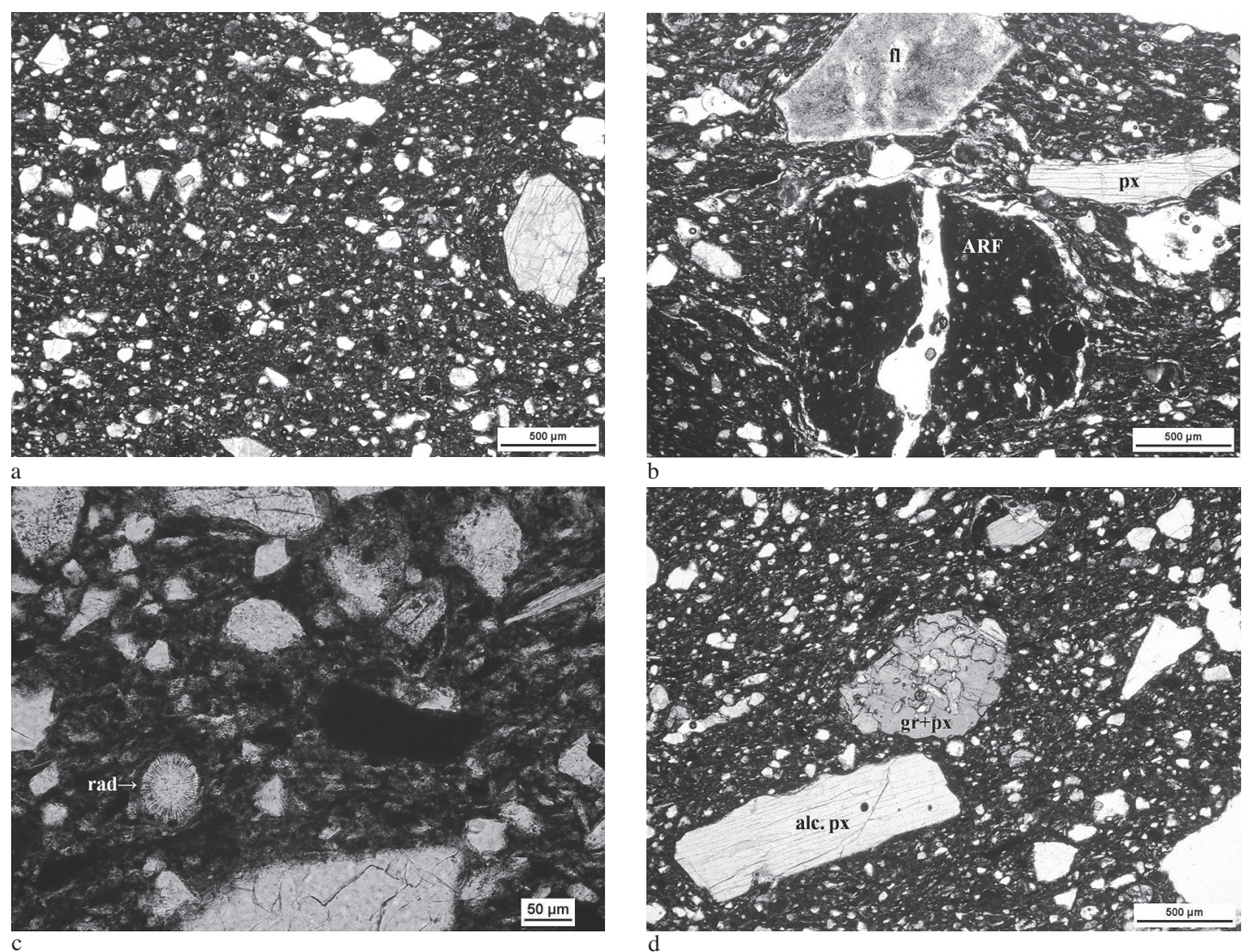

Fig. 1. Le immagini delle ceramiche appartenenti al I gruppo, eseguite con microscopio polarizzatore. a: Immagine generica della tessitura dei campioni del gruppo, vista con 1N; b: Selce (sopra, al centro), nucleo limonitico (sotto, al centro) e pirosseno alcalino (destra, al centro) vista con 1N (SP-8);

c: Immagine eseguita con microscopio polarizzatore di radiolaria (sferoidale, sotto. a sinistra) a $1 \mathrm{~N}$;

$\mathrm{d}$ : Immagine di pirosseno alcalino presente separatamente (forma di colonna allungata; angolo di sotto, a sinistra) e la geminazione del caratteristico granato verde giallastro con pirosseno pleocroico (sferoidale; al centro) realizzata con $1 \mathrm{~N}$

Il colore della matrice osservata al solo polarizzatore è rosso tendente al marrone o addirittura propriamente rosso (nel caso dei campioni SP-15 e SP-18) o rosso-marrone (SP-8, SP-17), e con nicol incrociati rosso molto scuro o eventualmente rosso tendente al marrone, (SP-8) oppure rosso-giallastro (SP-15). La struttura del campione SP-14 appartenente al gruppo I / b è a sandwich, eseguendo un'analisi con luce polarizzata a nicol paralleli il nucleo mostra un color marrone grigiastro mentre l'orlo ha un colore molto simile ai campioni SP-8 e SP-17 appartenenti al gruppo I / a, cioè rosso-marrone, e adottando nicol incrociati è isotropo. L'isotropia della materia prima del gruppo inteso in senso lato è varia, generalmente media, ma nel caso dei campioni SP-15 e SP-18 è scarsa. La matrice delle ceramiche SP-13, SP-14 e SP-17 è quasi interamente, o addirittura totalmente isotropa.

\section{Costituenti aplastici (>15 $\mu \mathrm{m})$ :}

Quarzo monocristallino: grandi quantità principalmente di granuli angolosi, piccoli e di medie dimensioni e prevalgono i granuli di estinzione ondulata.

Quarzo policristallino: granuli di granulometria media o grossolana. La quantità di questo materiale dipende dai granuli con granulometria media- o grossa. Non è presente nel campione SP-18. Generalmente è composto di individui cristallini e questi si collegano tramite contatto ondulato o, alcune volte tramite sutura. Nel campione S-18 sporadicamente è presente anche quarzo di filone.

Quarzite microcristallina (selce): presente generalmente in piccole quantità e manca completamente dal campione SP-3 (invece, questo campione contiene radiolarite). 

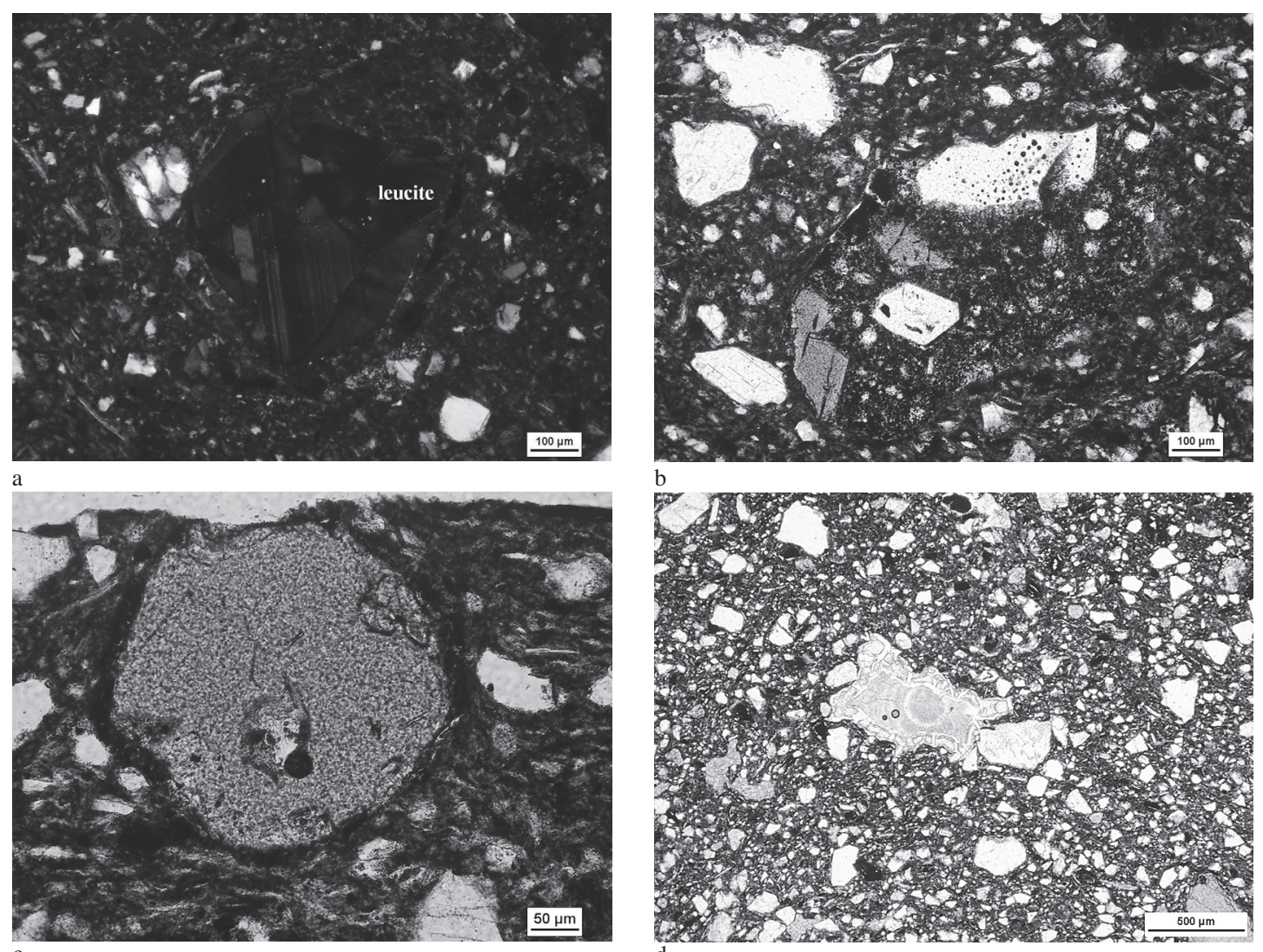
a

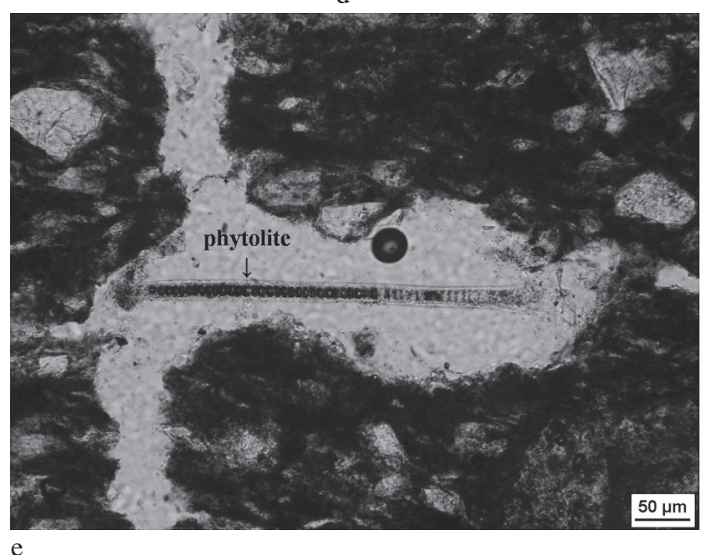

Fig. 2. a: Immagine della leucite eseguita con microscopio polarizzatore a N incrociati (SP-2); b: Tritume di vulcanite composto di pirossene alcalino, leucite e rocce vetrose, a $1 \mathrm{~N}$; c: Granato verde giallastro caratteristico per il gruppo, a 1N (SP-8); d: Tritume di opale e calcite visto con $1 \mathrm{~N}$ : immagine eseguita con microscopio polarizzatore di fitolite a $1 \mathrm{~N}-(\mathrm{SP}-8)$

Radiolarite/radiolari: è un costituente presente sporadicamente, l'abbiamo rilevato solo in due ceramiche (SP-3, SP-12).

Quarzite micacea: presente sporadicamente, l'abbiamo rilevato solo in due ceramiche (SP-2, SP-3) in forma di granuli di muscovite e quarzo poco arrotondati e di tessitura orientata,

$K$-feldspato: presente in ogni ceramica, ma in quantità modeste. In genere è idiomorfo, con struttura interna a mosaico o a strisce quindi non equigranulari. In alcuni campioni appare anche anortoclasio. 

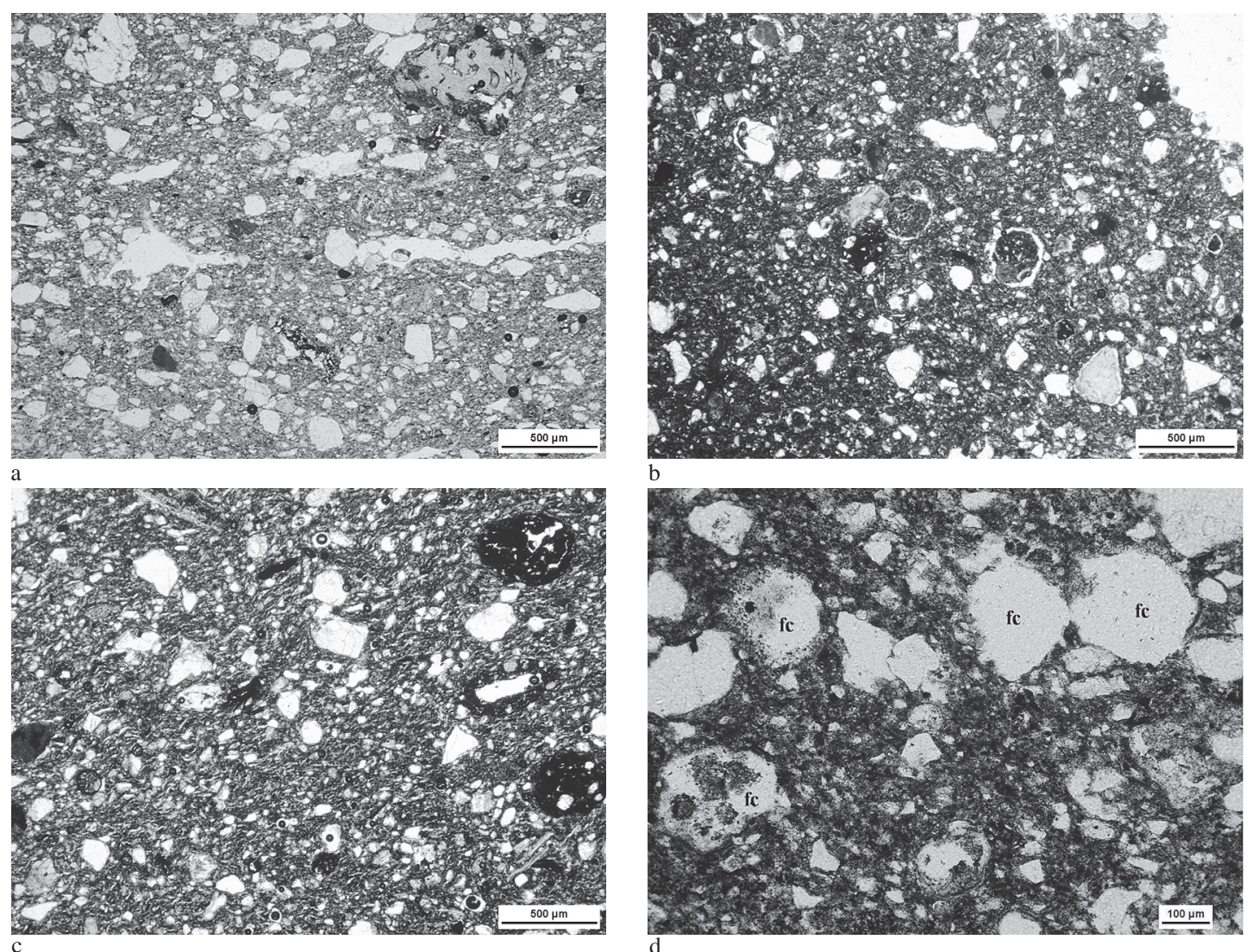

Fig. 3. Immagini delle ceramiche appartenenti al gruppo II eseguite con microscopio polarizzatore. a: Immagine della tessitura del campione SP-5 (pirosseno sopra, nell'angolo destro) vista con $1 \mathrm{~N}$;

b: Immagine della tessitura del campione SP-11 vista con 1N: Immagine della tessitura del campione SP-16 vista con 1N; $\mathrm{d}$ : Immagine della tessitura del campione SP-20 con pori post carbonato, bruciati, vista con $1 \mathrm{~N}$

Plagioclasio: in genere è presente in quantità piccole e differenti (generalmente la quantità è minore di quella del K-feldspato), comunque è presente in quasi ogni campione, in genere si tratta di granuli a tessitura zonata, riassorbiti aventi i bordi cresciuti ulteriormente. Spesso ricristallizza. Si trovano inoltre anche granuli arrotondati, molto sgretolati e frantumati.

Muscovite: piccolissime lamelle (deriva dall'argilla), il volume è piccolo, ma le scaglie stesse sono numerose o molto numerose, in alcuni casi la quantità numerica è media. Sono orientate quasi parallelamente rispetto alla parete della ceramica. In alcuni casi è stata trovata anche muscovite di maggiori dimensioni e di origini frammentarie.

Biotite: si tratta di un costituente presente sporadicamente, non è presente in ogni sezione sottile delle ceramiche. È di dimensioni maggiori della muscovite. Se n'è trovata una quantità relativamente grande nel campione SP-15. È più grande della muscovite, può essere di un colore vivace, ma spesso è incolore o limonitizzata.

Pirosseno: presente in una quantità relativamente importante idiomorfo-ipidiomorfo, verde, pleocroico (pirosseno alcalino), in base alle caratteristiche ottiche è composto da augite ed egirina, inoltre sono presenti pirosseni privi di pleocroismo (augite o augite diopsidica), in alcuni casi solo augite.

Contiene visibilmente granuli riassorbiti, in alcuni casi si rileva che deriva da rocce vulcaniche, infatti alcune volte è presente incluso in materiale oloialino (in roccia interamente vetrosa) sottile. Raramente al pirosseno alcalino e alla roccia vetrosa si accompagna anche leucite (SP-2), fenomeno questo che indica la medesima origine dei tre costituenti.

Leucite: in genere è presente in forma di granuli di grandi dimensioni, isometrici, quasi idiomorfi. È presente solo in alcune ceramiche (SP-2, SP-7, SP-18, SP-6). 

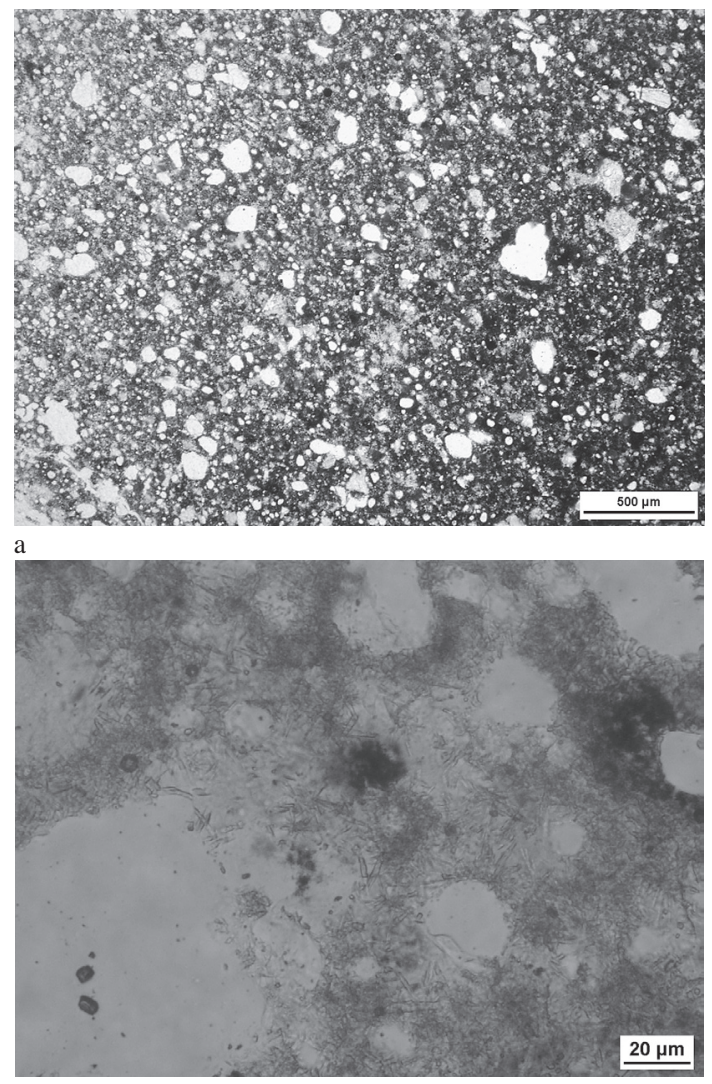

Fig. 4. Immagini delle ceramiche appartenenti al II gruppo eseguite con microscopio polarizzatore.

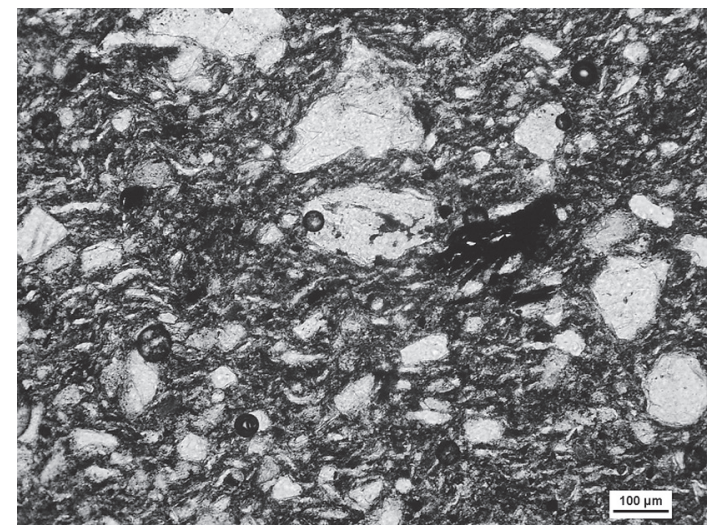

b

a: Immagine della tessitura del campione SP-21 con cavità amigdaloidi, vista con $1 \mathrm{~N}$; b: Immagine della tessitura del campione SP-16 striature rilevate nella materia prima, vista con $1 \mathrm{~N}$; c: La matrice composta di minerali aciculari vista con $1 \mathrm{~N}$ (SP-21);

$\mathrm{d}$ : Immagine generica tessiturale dei campioni appartenenti al gruppo vista con $1 \mathrm{~N}$

Minerali opachi: in ogni campione se ne rilevano quantità medie o piccole in forma di granuli piccoli, quasi isometrici. Inoltre, in alcune ceramiche compaino sporadicamente anche in quantità maggiori.

Minerali accessori: sono pochi, compaiono al massimo sporadicamente. I campioni contengono apatite, zircone, rutilo, titanite, epidote e granato incolore. (Nota: per via del particolare rilievo rivestito dal granato verde giallastro, abbiamo deciso di parlarne separatamente).

Granato verde-giallastro / granato associato a pirosseno: è di dimensioni relativamente grandi, di colore verde-giallastro. Spesso è presente assieme a pirosseno pleocroico, si può ipotizzare un'origine metamorfica di contatto (si compone di andradite e hedenbergite). Per l'esatta determinazione sarebbe necessario effettuare analisi in SEM-EDX.

Ne abbiamo trovate quantità piccolissime e non in ogni campione.

Frammenti di vulcanite-vulcanoclastite/roccia vetrosa (oloialina): materia prima costituita interamente o in gran parte da rocce vetrose contenenti micro-fenocristalli di pirosseno e minerali opachi (in questo caso contiene anche pochissimo e piccolissimo feldspato, pirosseno e minerali opachi). Sporadicamente si è potuto rilevare anche come sostanza di base la vulcanite composta esclusivamente di roccia vetrosa che, in tal caso, è variopinta, può essere marrone giallastra, marrone e nera. Raramente è presente in forma di cavità amigdaloide (SP-3). In uno dei campioni (SP-17) abbiamo rinvenuto tritume di opale e calcite che, probabilmente, deriva da vulcanoclastite. Questo materiale è presente in quantità variabile e in alcuni casi è totalmente assente.

Frammenti di granitoide: compaiono saltuariamente, è presente solo nella ceramica SP-14. È costituito dall'intercrescita di feldspato e quarzo. 

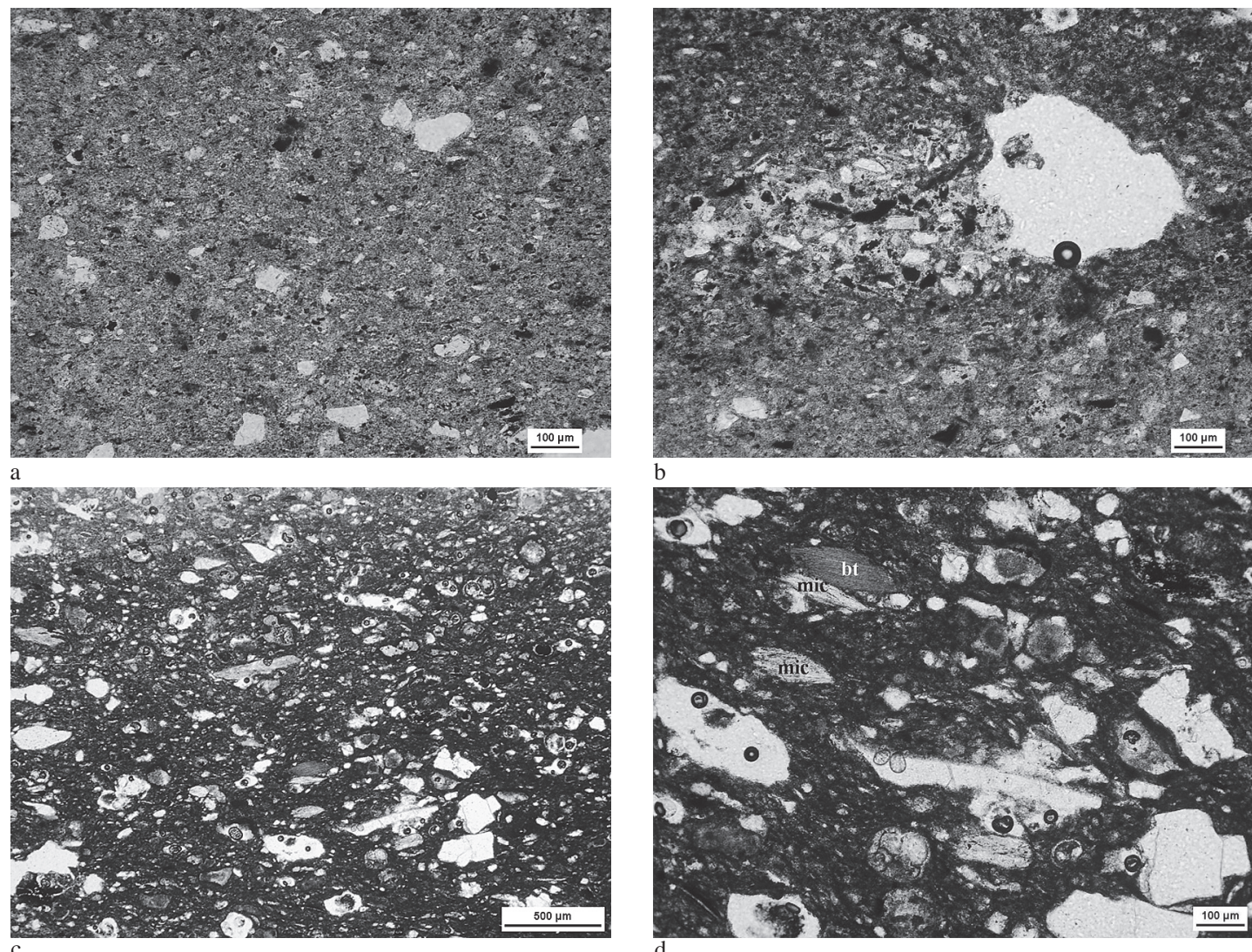

$\mathrm{b}$

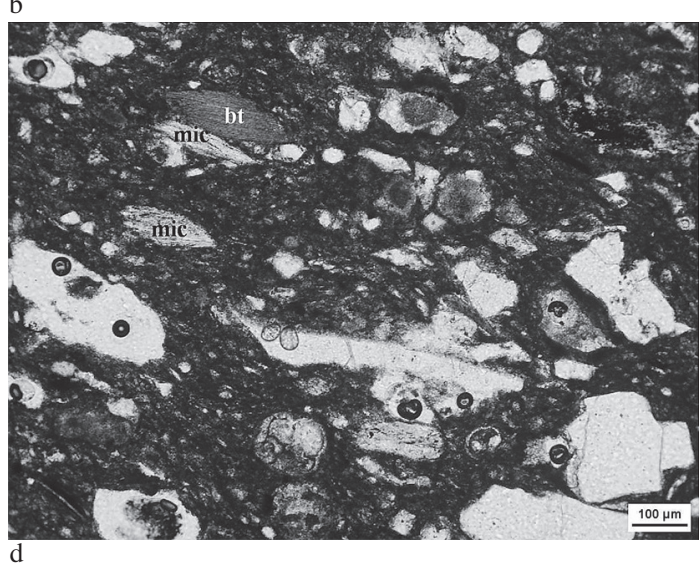

Fig. 5. Immagini delle ceramiche appartenenti al gruppo III eseguite con microscopio polarizzatore.

a: Pseudomorfi limonitici post biotite e post magnetite a 1N (SP-10); b: Abbondanza di frammenti fini che fa supporre l'utilizzo di una miscela di argilla (SP-10). Immagini delle ceramiche appartenenti al gruppo IV eseguite con microscopio polarizzatore; c: Immagine generica della tessitura del campione appartenente al gruppo vista con $1 \mathrm{~N}$; d: Biotite (color marrone) e sotto due aggregati di mica incolore visti con $1 \mathrm{~N}$

Frammenti di calcare: il carbonato standard primario è presente in piccolissime quantità, esclusivamente nei campioni SP-8 e SP-15. Nel primo un granulo di dimensioni maggiori (di $\sim 1 \mathrm{~mm}$ ) nel secondo si rilevano alcuni piccoli granuli arrotondati di micrite.

\section{Altri costituenti.}

Noduli di argilla limonitica / frammenti di rocce argillose $(A R F)$ : presente in quasi ogni ceramica in quantità differenti. Non contiene per nulla, o solo in piccolissime quantità, granuli isometrici e sferoidali. Nella stragrande maggioranza le ceramiche contengono noduli argillosi limonitici. In uno dei campioni (SP-15) il nodulo di argilla limonitica è accompagnato da frammenti di roccia argillosa (ARF).

Resti vegetali carbonizzati: è un costituente presente solo nel campione SP-14 (sandwich). Si tratta di granuli frantumati che indicano lo smagramento con vegetali.

Fitoliti: esclusivamente nel campione SP-8 compare un unico granulo allungato.

\section{Interpretazione (I/a):-}

La materia prima delle ceramiche è costituita da argilla / miscele di argilla prive di calcare contenenti scheletro di grana medio-fine, probabilmente smagrata intenzionalmente. In vari campioni di ceramica compaiono frammenti di roccia argillosa (ARF) limonitizzati. La presenza di tali materiali pare indicare che per la produzione furono adottate argille differenti; infatti, la materia prima può essere costituita dalla miscela di una materia più grassa e di una più magra, lo attesta da una parte il campione SP-8 che contiene una quantità rilevante di dimensioni 

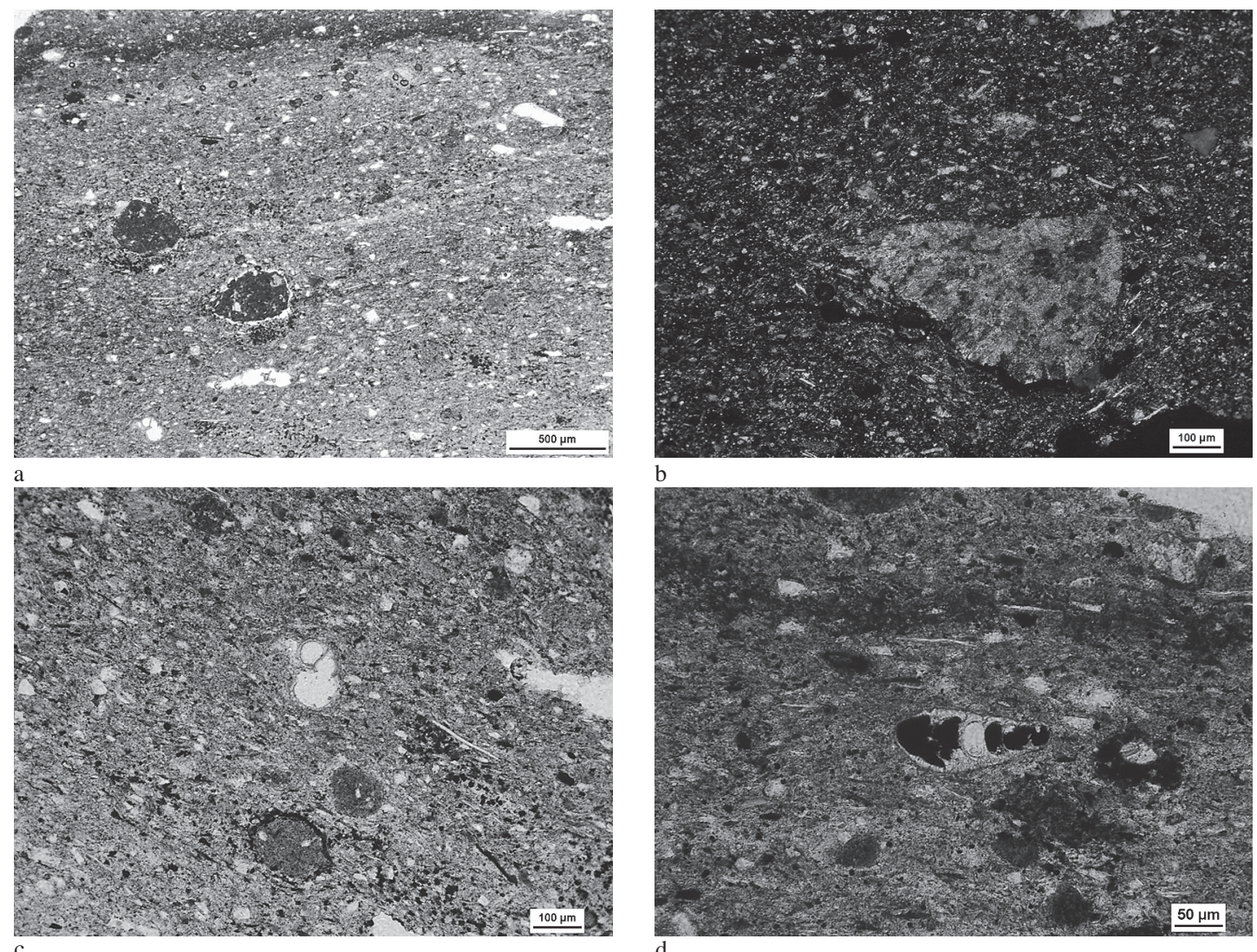

Fig. 6. Immagini delle ceramiche appartenenti al gruppo $\mathrm{V}$ eseguite con microscopio polarizzatore.

a: Immagine generica della tessitura del campione appartenente al gruppo, vista con 1N; b: Granuli di micrite scarsamente arrotondati, visti con 1N; c: Reperti fossili con vari ventricoli, probabilmente di tipo Globigerinoides visti con 1N (SP-9); d: Reperti fossili con vari ventricoli, con riempimento di minerale opaco (SP-9)

considerevoli di noduli di argilla limonitizzata di origine ARF. D'altra parte, invece, il campione SP-15 che, a sua volta contiene pure una considerevole quantità di ARF, comunque è stato fabbricato con una materia prima più grassa delle altre, fatto questo convalidato anche da un costituente aplastico di quantità e dimensioni minori.

Tra i costituenti aplastici vanno evidenziati i costituenti alcalini di origine vulcanica, presenti in quantità relativamente rilevante come granuli di dimensioni considerevoli (idiomorfi, di pirosseno alcalino verde pleocrooico, leucite e materiale di base vulcanico di cui si trova anche una variante composta di vetro puro, nonché roccia vetrosa e cristalli). Sporadicamente si può rilevare un insieme composto di fenocristalli pirossenici con bordo di materiale di base vulcanico sottile, a cui in un unico campione si unisce anche leucite. Ciò indica l'uso di materiale smagrante alcalino insaturo, di origine vulcanica. L'aggiunta di degrassante è segnalata anche dal carattere iatale delle ceramiche. In alternativa, che però non è molto verosimile, si può ipotizzare anche che la materia prima delle ceramiche provenisse da una cava nelle cui vicinanze ci fossero rocce alcaline, di origine vulcanica, contenenti pirosseno e leucite e l'argilla veniva estratta nei pressi di quest'ultime.

Le ceramiche furono cotte in ambiente ossidante e la temperatura di cottura nel caso delle ceramiche con matrice leggermente isotropa probabilmente non era più alta di $650-700{ }^{\circ} \mathrm{C}$, mentre per le ceramiche isotrope o quasi (SP-13, SP-17 e SP-14 appartenente al gruppo I / b) la temperatura poteva essere più alta, oltrepassando anche i $750-800^{\circ} \mathrm{C}$. In conformità a ciò di cui sopra le condizioni di cottura dei campioni del gruppo I sono molto varie.

\section{Altre osservazioni:}

Il campione SP-18 è molto limonitizzato e in disfacimento probabilmente perché è stato cotto a temperature troppo basse o perché è rimasto a lungo in ambiente corrosivo (per es. acido e umido). 
Le ceramiche SP-2 e SP-17 sono rivestite all'esterno di carbonato di calcio. Nel campione SP-17 si rilevano macchie di impregnazione con carbonato a grana fine (i pori sono riempiti di calcite a grana fine) rivelando che questo campione dovette subire carbonatazione secondaria.

Le differenze minori che si rilevano tra le caratteristiche dei campioni SP-15 e SP-18 rivelano che sono state fabbricate con una materia prima uguale ma in condizioni tecnologiche alquanto differenti, però questa osservazione non giustifica a nostro avviso la creazione di un sottogruppo separato.

\section{Gruppo I / b)}

I costituenti clastici dei campioni facenti parte del sottogruppo e la matrice argillosa sono molto simili a quelli delle ceramiche del gruppo I / a. La differenza sta nella quantità dei granuli clastici che nel secondo gruppo è maggiore del primo e invece i pirosseni alcalini idiomorfi di grandi dimensioni sono sostituiti da granuli simili, ma più fini e frantumati maggiormente, cioè il tritume di origini vulcaniche è di carattere ridepositato e più maturo. Questo fenomeno è attestato anche dalla maggior quantità di quarzo e minor quantità di feldspati ritrovati nelle ceramiche.

Si desidera evidenziare anche la struttura a sandwich della ceramica SP-14. All'interno della ceramica si sono conservati resti di materie organiche bruciate (carbone). Assieme ai pori di dimensioni relativamente grandi, osservati nelle altre ceramiche questo fenomeno potrebbe indicare che le ceramiche del gruppo I furono prima cotte in ambiente riducente e poi il raggiungimento e il mantenimento della temperatura massima dei materiali è avvenuto in ambiente ossidante. Nel caso del campione SP-14 o la conservazione del materiale alla temperatura massima di cottura era più breve o la quantità del materiale organico aggiunto alla miscela era maggiore e questo ha conservato la struttura a sandwich.

\section{Gruppo I (generico)}

Il campione SP-13 è molto piccolo e mal conservato e ciò influisce sulla quantità dei singoli costituenti, anzi certi costituenti comunque presenti in piccole quantità possono eventualmente sparire. Per questa ragione senza classificarlo più precisamente, abbiamo inquadrato genericamente questo campione nel gruppo I.

\section{Gruppo II}

(Fig. 3-4.)

Composto di cinque ceramiche: SP-5, SP-11, SP-16, SP-20, SP-21

\section{Descrizione macroscopica.}

Il colore delle ceramiche di questo gruppo è variabile, va dal color mattone al grigio, dipendendo dalle condizioni in cui si è svolta la cottura. Le ceramiche SP-5 e SP-21 sono a grana fine, nei tre campioni rimanenti si rilevano, similmente al gruppo I, piccoli granuli bianchi, neri e micacei.

\section{Descrizione microscopica.}

Tessitura

In base alla granulometria dei costituenti aplastici la tessitura è seriale o leggermente iatale, fatta eccezione per il campione SP-21 che è certamente di tessitura iatale. In genere i campioni sono caratterizzati da due intervalli di granulometria dominanti che vanno da (15 -)20-50(-60) $\mu \mathrm{m}$ e da $\mathbf{1 0 0}$ a $\mathbf{3 0 0} \mu \mathrm{m}$, mentre il massimale relativo alla granulometria dei campioni, salvo quella SP-21, in cui il massimale raggiunge solo i $250 \mu \mathrm{m}$, è di 500-700 $\mu \mathrm{m}$. La tessitura delle ceramiche di questo gruppo è poco o scarsamente classata, mentre quella del campione SP-21 è molto ben classata. I granuli generalmente non sono arrotondati, o sono appena un po' arrotondati, il campione SP-16 contiene molti granuli frantumati, angolosi.

La proporzione dei costituenti aplastici, le cui dimensioni superano i $15 \mu \mathrm{m}$ è, in genere, di circa del $20 \%$. Il campione SP-16 ne contiene di più (25\%), mentre la quantità contenuta nel campione SP-21 è molto più bassa, è del 6\%.

La tessitura delle ceramiche è moderatamente compatta con pochi pori irregolari, raramente allungati (SP-5). Invece nel campione SP-21 i pori sono sferoidali con dimensioni che non superano i $100 \mu \mathrm{m}$ e la tessitura del campione SP-16 è molto compatta, i pori non sono visibili neanche al microscopio. 
Il colore della matrice osservata a nicol paralleli è vario, in genere giallo-rossastro mediamente scuro, rosso tendente al marrone, marrone o marrone grigiastro, con nicol incrociati, salvo il campione SP-5, isotropo. Il colore variegato indica, per l'appunto, una minore variabilità delle condizioni di ossidazione e riduzione subite dai materiali durante la cottura.

Costituenti aplastici (>15 $\mu m)$ :

Quarzo monocristallino: presenza dominante, se ne rilevano grandi quantità in granuli di estinzione ondulata con granulometria piccola e media.

Quarzo policristallino: presente in genere tra i tritumi di granulometria maggiore in quantità relativamente piccola.

Quarzite microcristallina (selce): presente sporadicamente, in piccolissime quantità e manca completamente dal campione SP-21.

$K$-feldspato: presente in tutte le ceramiche, ma in quantità moderate. In genere è idiomorfo, variegato e raramente vivace. Inoltre compaiono in esso granuli di perlite con struttura interna a mosaico, non equigranulare. In alcuni campioni è presente anche anortoclasio.

Plagioclasio: presente in quantità minori rispetto al feldspato di potassio, spesso la quantità è piccolissima, ma si ritrova in ogni ceramica.

Muscovite: presente in piccole o medie quantità nella matrice con granulometria generalmente molto fine, ma alcune volte si ritrovano anche granuli più grossolani, di origine detritica.

Biotite: presente solo in tre ceramiche (SP-11, SP-16, SP-20), nel campione SP-11 in quantità media, mentre negli altri due campioni se ne trova ben poca. Spesso è alterata in limonite.

Pirosseno alcalino: composto di granuli di varie dimensioni che, prevalentemente sono però piccoli e frammentari, mentre i granuli più grandi, idiomorfi, simili a quelli del gruppo I, sono presenti solamente sporadicamente. La quantità di questo minerale è comunque piccola, compare spesso solo in modo saltuario, 1-2 granuli nelle singole ceramiche.

Materia prima vulcanica: non appare in ogni campione, è presente occasionalmente o in piccolissime quantità. In genere contiene piccoli costituenti cristallini, ma nel campione SP-20 è composta esclusivamente di rocce vetrose, oloialine.

Micascisto/scisto cristallino: presente sporadicamente, solo in due ceramiche (SP-5, SP-11).

Minerali opachi: granuli generalmente di piccole dimensioni, quasi isometrici, presenti in minime quantità. Solo nel campione SP-16 si rilevano granuli più grossolani.

Minerali accessori: pur essendo presenti in varie quantità, tuttavia li ritroviamo in ogni campione (clinozoisite, zoisite, ortite, zircone, tormalina, granato (incolore), rutilo.

Granato verde giallastro: presente sporadicamente in tre ceramiche (SP-5, SP-16, SP-20), simile al tipo verde giallastro rilevato nel gruppo I, con la differenza che qui i granuli sono più piccoli e clastici.

Frammenti di granitoide: presente sporadicamente nella forma di geminazione di plagioclasio e quarzo.

Frammenti di calcare: nel campione SP-11 si rileva una quantità media di granuli abbastanza grandi ben arrotondati e micritizzati, in parte carbonizzati (forse appartenne a questo contesto anche un reperto fossile carbonatico; inoltre, il tritume è presente molto raramente anche in forma di granuli piccolissimi. Nel campione SP-20 sono presenti frantumi micritizzati di roccia calcarea, fortemente carbonizzati in tempi molto remoti. Nel campione SP-5 non si trova carbonato e non doveva esserci nemmeno originariamente, mentre per quel che riguarda il campione SP-21, essendo questo molto ben cotto e carbonizzato, non si può stabilire se originariamente contenesse o no carbonato primario.

Reperti fossili: di forma sferoidale.

Senza dubbio presenti in quantità media nel campione SP-20, nonostante il carbonato sia in maggior parte incenerito. Saltuariamente contengono riempimenti di minerali opachi.

\section{Altri costituenti:}

Noduli argillosi-limonitici sferoidi, di 150-300 $\mu \mathrm{m}$, nonché tritume argilloso di rocce ARF con all'interno tritume di piccole dimensioni. Raramente sono presenti anche in forma considerevolmente più grande. 


\section{Interpretazione:}

La ragione dell'inquadramento delle ceramiche nello stesso gruppo è la presenza di certi costituenti aplastici (per es. pirosseno alcalino, feldspati di forma simile, una quantità notevole di quarzo). Questi costituenti sono simili a quelli presenti nel gruppo I, ma generalmente sono più frammentari e più piccoli. Ciò indica che il materiale smagrante è stato frantumato, oppure proviene da un sito più distante dalla fonte vulcanica.

Il materiale plastico di questo gruppo è molto vario, generalmente è stata adottata argilla piuttosto grassa, priva di carbonati. In base al tritume di carbonato, contenente micrite e/o ai resti fossili contenenti carbonato, si può ipotizzare che al materiale fosse stata aggiunta argilla contenente carbonato di calcio o marna (in base ai reperti fossili con riempimenti di minerali opachi questi potevano essere di origine flysch / fluida, cioè derivanti da rocce sedimentarie clastiche) e che lo smagramento fosse stato eseguito con l'aiuto di sabbia contenente carbonati.

Le ceramiche in questione subirono effetti termici molto diversi. La diversità può derivare dalle differenze delle condizioni della cottura a cui le ceramiche vennero sottoposte, o da effetti termici posteriori e questa varietà si manifesta nella differenza delle caratteristiche tessiturali macroscopiche e microscopiche. L'isotropia scarsa della matrice rivela che, la temperatura di cottura della ceramica SP-5 fabbricata con materia prima grassa, priva di carbonati, dovette essere relativamente bassa, inferiore a $700{ }^{\circ} \mathrm{C}$. Infatti, la temperatura di cottura delle ceramiche con matrice isotropica è di $750-800^{\circ} \mathrm{C}$, o anche più alta. Quest'ultima constatazione può essere riferita anche al campione SP-16, in cui si rilevano striature causate probabilmente dalla plasticità derivante dalla cottura eseguita a una temperatura molto alta $\left(900{ }^{\circ} \mathrm{C}\right)$. La diversità delle caratteristiche del campione SP-21 rispetto a quelle degli altri campioni è dovuta all'effetto termico straordinariamente alto che questo materiale ha subito (o forse all'uso? o a combustione posticipata?), e che ha risultato cavità amigdaloidi; la matrice, invece, è composta di piccolissimi minerali sottili, situati senza orientazione, forse di pirosseno. Comunque, il fatto che i granuli clastici di ambedue i campioni siano molto simili a quelli delle altre ceramiche ci ha indotti a inquadrarli in questo gruppo.

\section{Gruppo III}

(Fig. 5.)

Composto da due ceramiche: SP-10, SP-19

\section{Descrizione macroscopica}

Ceramiche a grana fine, bianca, a occhio nudo non si possono distinguere costituenti clastici.

\section{Descrizione microscopica}

\section{Tessitura}

In base alla granulometria dei costituenti aplastici la tessitura delle ceramiche è seriale.

La granulometria dominante dei costituenti aplastici è in generale pari a 15-40 $\mu \mathrm{m}$. Nel campione SP-10 la dimensione massima dei granuli è di $\mathbf{3 8 0} \mu \mathrm{m}$, nel campione SP-19 $200 \mu \mathrm{m}$ (ambedue le ceramiche contengono solo saltuariamente granuli clastici più grandi di $100 \mu \mathrm{m})$. La classazione delle ceramiche è buona. I granuli sono scarsamente arrotondati, ma nel campione SP-9 i granuli di dimensione più grossolana sono maggiormente arrotondati.

Nel caso di ambedue le ceramiche, la percentuale dei costituenti aplastici più grandi di $15 \mu \mathrm{m}$ è pari a circa il $3 \%$.

Le ceramiche sono molto compatte, nel campione SP-10 si rilevano alcuni pori di forma irregolare, di dimensioni relativamente grandi $(250-800 \mu \mathrm{m})$, orientati parallelamente rispetto alla parete della ceramica. Nel campione SP-19 i pori la cui dimensione dominante è piuttosto piccola (100-300 $\mu \mathrm{m})$ sono rari e non mostrano nemmeno orientazione.

Il colore della matrice in ambedue i campioni visto con 1 nicol è bruno grigiastro, con nicol incrociati è isotropo.

Costituenti aplastici ( $>15 \mu \mathrm{m})$ :

Quarzo monocristallino: nei campioni si rileva una quantità dominante di granuli di estinzione normale e ondulata in proporzione di 1:1. I granuli di dimensioni piuttosto grandi sono poco- o mediamente arrotondati.

Quarzo policristallino: presente solo nel campione SP-10 in quantità ridotta, con granuli piuttosto grossi. 
Quarzite microcristallina (selce): presente sporadicamente in ambedue i campioni in granuli ben arrotondati e relativamente grandi. Inoltre nel campione SP-10 in un granulo di selce abbiamo osservato la presenza di un radiolare.

K-feldspato: presente saltuariamente e solo nel campione SP-10.

Plagioclasio: presente solo nel campione SP-19, un granulo di dimensioni piuttosto grandi.

Muscovite: presente in piccole quantità nella forma di lamelle piccolissime orientate parallelamente rispetto alla parete della ceramica.

Pseudomorfosi limonitiche: presenti in quantità piuttosto notevoli su piccoli individui di biotite e magnetite, costituenti minuti, limonitizzati post-biotite (sezioni allungate) e post-magnetite (sezioni isometriche).

Reperti fossili: presenti in ambedue i campioni quantità medie di resti fossili con riempimenti limonitici, nonché relitti degli stessi. Nel campione SP-19 sono chiaramente riconoscibili come globigerinoidi, mentre nel SP-10 sono maggiormente trasformate e quindi si presentano come noduli ovoidali o sferoidali con riempimenti limonitici.

\section{Interpretazione}

Le due ceramiche del gruppo III sono di grana molto fine, fabbricate originariamente direttamente con argilla siltosa calcarea (miscelata con silt) contente pochi granuli clastici, o con una miscela di argilla, o forse prodotta utilizzando il materiale decantato della stessa. In certe parti i frammenti fini abbondano, il che fa supporre l'utilizzo di una miscela di argilla. La presenza delle Globigerinoides con riempimenti di materiale limonitico nella materia prima di queste ceramiche, permette di ipotizzare che sia completamente o in parte di origine da un flysch. Comunque per via dell'alta temperatura di cottura, probabilmente $>900^{\circ} \mathrm{C}$, il carbonato è stato completamente distrutto.

\section{Gruppo IV}

A questo gruppo appartiene solo una ceramica: SP-1

\section{Descrizione macroscopica.}

Originariamente la ceramica doveva essere di color rosa pallido, ma la superficie si è scolorita, probabilmente a causa di una alterazione post-deposizionale.

L'impasto è a grana fine, a occhio nudo non si vedono granuli clastici.

\section{Descrizione microscopica.}

\section{Tessitura:}

In base alla granulometria dei costituenti aplastici contenuti nella ceramica, questa è seriale, se però si considerano solo i costituenti con carbonato, allora la tessitura è iatale con distribuzione bimodale. La granulometria dominante della frazione fine dei costituenti aplastici è generalmente di $\mathbf{2 0 - 4 0} \mu \mathrm{m}$, mentre la frazione con grana più grossolana è in genere di 100-150 $\mu \mathrm{m}$, ma si possono rilevare anche granuli che si collocano tra i due intervalli dimensionali. Il massimale della granulometria raggiunge i $\mathbf{3 0 0} \mu \mathrm{m}$. Le inclusioni sono mediamente classate, i granuli in genere non sono arrotondati o sono di arrotondamento scarso, raramente si rilevano anche granuli ben arrotondati.

La percentuale dei costituenti aplastici di dimensioni che superano i $15 \mu \mathrm{m}$ è pari al $15 \%$, di cui $10 \%$ contiene carbonato e ne è privo solo il $5 \%$.

La ceramica è molto compatta, vi si rilevano pochi pori di forma irregolare (generalmente la dimensione è di $250-500 \mu \mathrm{m}$ ) e si possono osservare anche pori creatisi in sostituzione dei costituenti dissociati che contenevano carbonato.

Il colore della matrice visto a nicol sia paralleli, sia incrociati è grigio scuro. L'isotropia della matrice è media.

\section{Costituenti aplastici $(>15 \mu \mathrm{m})$ :}

Quarzo monocristallino: presente in ogni classe dimensionale, granuli di estinzione ondulata in quantità relativamente grandi.

Quarzo policristallino: poco, principalmente si osservano granuli di dimensioni grossolane che sporadicamente possono essere associati a miche. 
Quarzite microcristallina (selce): presente sporadicamente nel campione.

Muscovite: se ne osservano alcune piccole lamelle.

Biotite: una quantità media di granuli più grandi di quelli della muscovite in stato fortemente limonitizzato, a causa della $\mathrm{T}^{\circ}$ di cottura.

Aggregati di mica incolori: presente una quantità relativamente ridotta di noduli un po' più grandi, costituiti da muscovite tuariamente.

Micascisto: i clasti sono composti da quarzo a grana fine e mica bianca a struttura orientata, presente saldimensioni.

Minerali opachi: presenti pochi e piccoli granuli quasi isometrici, accompagnati da alcuni granuli di medie

Frammenti di calcare: sono presenti in grandi quantità frammenti carbonatici ben arrotondati, di dimensioni medio-grandi, micritici.

Reperti fossili: presenti principalmente resti fossili (Globigerinoidi) di carbonato completamente dissociati con riempimenti limonitici sparsi.

\section{Altri costituenti:}

Sporadicamente compaiono noduli limonitici-argillosi di piccole dimensioni.

\section{Interpretazione:}

La materia prima della ceramica era probabilmente composta da flysch calcareo alla quale probabilmente fu aggiunta sabbia, ma si può supporre anche un miscuglio di argilla calcarea a grana fine e argilla più magra contenente una frazione di dimensioni sabbiose. Per la cottura si può stimare una temperatura attorno ai $750^{\circ} \mathrm{C}$.

Per quel che riguarda gli inclusi aplastici, il campione somiglia a quello indicato col numero SP, ma contenendo una quantità considerevole in più di granuli clastici grossolani, si è deciso di inquadrarlo in un gruppo separato.

\section{Gruppo $V$ \\ (Fig. 6.)}

Questo gruppo contiene una ceramica: la SP-9

\section{Descrizione macroscopica.}

Le ceramiche di questo gruppo sono di colore rosso mattone con all'interno piccoli granuli bianchi e neri, nonché piccole particella di mica.

\section{Descrizione microscopica.}

Tessitura:

La tessitura della ceramica in base alla granulometria dei costituenti aplastici in essa contenuta è iatale con distribuzione bimodale a 2 massimali. La granulometria dominante della frazione fine dei costituenti aplastici è generalmente di 15-30 $\mu \mathrm{m}$, mentre la frazione con grana più grossolana è in genere di 100-150 $\mu \mathrm{m}$. Il massimale della granulometria raggiunge gli $\mathbf{8 5 0} \mu \mathrm{m}$, ma questa dimensione è rappresentata solo da un unico granulo. La ceramica è molto ben classata. I granuli piuttosto grandi in genere non sono arrotondati o solo scarsamente, in qualche raro caso possono essere anche mediamente arrotondati.

La percentuale dei costituenti aplastici di dimensioni maggiori di $15 \mu \mathrm{m}$ è circa del 3\%. La ceramica è relativamente compatta, però si possono osservare in essa molti micro pori.

Il colore della matrice osservato ad 1 nicol è marrone tendente al giallo chiaro, con nicol incrociati è marrone grigiastro. In tutta la matrice è diffusa calcite microcristallina a grana fine, per cui osservata a nicol incrociati mostra una tessitura a pallini. L'isotropia della materia prima è molto scarsa. 


\section{Costituenti aplastici $(>15 \mu \mathrm{m})$ :}

Quarzo monocristallino:presente in grandi quantità principalmente nella categoria con granulometria fine, ma sporadicamente se ne trova un po'anche nella frazione dimensionale grossolana. I granuli di dimensioni minori sono di estinzione ondulata, i più grandi ad estinzione normale (forse sono di origine vulcanica).

Quarzo policristallino: presente una piccola quantità tra i costituenti con granulometria piuttosto grossolana. della ceramica.

Muscovite: è dominante la presenza di scaglie piccolissime, orientate parallelamente rispetto alla parete (50-60 $\mu \mathrm{m})$.

Biotite: costituente presente saltuariamente con granuli di dimensioni più grandi di quelle della muscovite

Clinopirosseni: presenti sporadicamente, in granuli di dimensioni piuttosto grandi.

Minerali opachi: presenti in grandi quantità in piccolissimi granuli quasi isometrici. Qua e là si uniscono in aggregati e mostrano tendenza alla limonitizzazione.

Tritume di calcare: presenti vari granuli di piccole dimensioni e un granulo di micrite più grande.

Reperti fossili: presenti in forma di resti fossili di tipo Globigerinoides, con due o più ventricoli ben visibili. Raramente contengono riempimenti di minerale opaco, inoltre spesso si rilevano anche frammenti di guscio. Ambedue i reperti fossili sono composti di carbonato.

\section{Altri costituenti:}

Frammenti di rocce argillose $(A R F)$ : sferoidali, ben arrotondati, di color rosso-bruno. Le dimensioni medie sono di 100-150 $\mu \mathrm{m}$, al massimo raggiungono i $400 \mu \mathrm{m}$. Contengono saltuariamente piccoli granuli, in maggior parte di quarzo.

\section{Interpretazione:}

Il materiale che costituisce le ceramiche è in parte calcare (in base ai reperti fossili si può supporre che fosse originariamente un flysch) che, come lo attesta la notevole quantità di mica, dovette essere mischiato ad argilla grassa. Da quest'ultima provengono anche alcuni granuli clastici grossolani. In base alla presenza dei carbonati e la scarsa isotropia della matrice si suppone che la temperatura di cottura della ceramica dovette essere bassa, inferiore a $700^{\circ} \mathrm{C}$. In base alla presenza del calcare e dei fossili, somiglia parzialmente alle ceramiche del gruppo III, ma la matrice molto micacea indica una similitudine alle ceramiche del gruppo I. Questa è la ragione per cui abbiamo deciso di inquadrare in un gruppo separato questa ceramica.

\section{BIBLIOGRAFIA}

PCRG 1997

PETTIJohn e altri 1972

QuinN 2013

SZAKMÁNY 1996

SZAKMÁNY 1998

SZAKMÁNY 2001

TUCKER

WHITBREAD 1986

WHITBREAD 1989
= The Study of Later Prehistoric Pottery: general policies and guidelines for analysis and publication. Prehistoric Ceramic Research Group: Occasional Papers 1 and 2. 1997.

= F. J. Pettijohn-P. E. Potter-R. Siever: Sand and Sandstone. Springer-Verlag, New York 1972.

= P. S. QuinN: Ceramic Petrography. Archaeopress-Gordon House, Oxford 2013.

$=$ Gy. SZAKMÁNY: Petrographical investigation in thin section of some potsherds. In: J. Makkay-E. Starnini-M. Tulok: Excavations at Bicske-Galagonyás. 3: The Notenkopf and Sopot-Bicske cultural phases. Società per la Preistoria e Protostoria della Regione Friuli-Venezia Giulia, Quaderno 6. Trieste 1996, 143-150.

$=$ GY. SZAKMÁNY: Insight into the manufacturing technology and the workshops: evidence from petrographic study of ancient ceramics. In: L. Költő-L. Bartosiewicz (eds): Archaeometrical Research in Hungary II. Budapest 1998, 77-83.

= Gy. SZAKMÁNY: Felsővadász-Várdomb neolitikus és bronzkori kerámiatípusainak petrográfiai vizsgálata [Analisi petrografica dei tipi di ceramica neolitica e dell'età del bronzo rinvenuti nella collina Felsővadász-Várdomb]. Herman Ottó Múzeum Évkönyve [Annuario del Museo Ottó Herman di Miskolc] 40 (2001) 107-125.

= M. TUCKER: Sedimentary Petrology. Blackwell, Oxford 2001.

$=\mathrm{I}$. K. WhITBREAD: The characterisation of argillaceous inclusions in ceramic thin sections. Archaeometry 28 (1986) 79-88.

= I. K. WhitBREAD: A proposal for the systematic description of thin sections towards the study of ancient ceramic technology. In: Y. Maniatis (ed.): Archaeometry. Proceedings of the $25^{\text {th }}$ International Symposium. Elsevier, Amsterdam 1989, 127-138. 